DOI: 10.17234/SRAZ.65.58

UDK: 821.163.42.09 Gundulić, I.

UDK: 821.131.1.09 Rinuccini, O.

UDK: 821.131.1-293.09

UDK: 811.131.1'255.4=163.42

Original scientific paper

Ricevuto il 13 luglio 2020

Approvato per la pubblicazione il 25 novembre 2020

\title{
Gundulić traduttore di Rinuccini. Analisi della versione croata del Lamento di Arianna
}

\author{
Sanja Roić \\ Katja Radoš-Perković \\ Università di Zagabria \\ sroic@ffzg.hr \\ krperkov@ffzg.hr
}

La relazione prende in esame la traduzione croata di un libretto d'opera di Ottavio Rinuccini, l'Arianna, entrato nella storia della musica grazie all'apporto musicale del primo grande compositore d'opera Claudio Monteverdi che l'ha musicato e messo in scena nel 1608. La traduzione croata viene allestita molto precocemente, già nel 1615, e pubblicata nel 1633. L'autore dell' Arijadna è Ivan Gundulić, il maggior rappresentante del barocco croato, che nella sua carriera poetica si dedicherà diverse volte a traduzioni e adattamenti di libretti d'opera italiani. Nell'indagine verranno messi a confronto i rispettivi testi italiano e croato del Lamento di Arianna (il frammento più conosciuto di questo libretto in quanto il solo di cui si conserva lo spartito musicale monteverdiano) con l'obiettivo di determinare quali sono stati i procedimenti traduttivi che Gundulić aveva impiegato e con quali esiti nel testo croato. Attraverso esempi concreti verranno presentate le scelte stilistiche e lessicali del traduttore e i suoi interventi originali (sottoforma di aggiunte, spiegazioni o altro) che furono possibili perché il libretto, da quello che le ricerche hanno rilevato, veniva rappresentato come dramma recitato e non come dramma per musica (opera lirica).

Parole chiave: Gundulić, Rinuccini, Arianna, traduzione, Zore

\section{Introduzione}

Dopo aver compiuto gli studia humanitatis nella città nativa sotto la guida di Camillo Camilli, ottimo conoscitore di Tasso, e del maestro Petar Palikuća, Ivan Gundulić inizia la propria attività letteraria intorno al 1610. Suo padre, ben cinque volte eletto rettore della Repubblica di Dubrovnik, aveva compiuto diversi viaggi nelle città lontane, Roma, Costantinopoli e Napoli, di cui sicuramente aveva raccontato al figlio. Nel 1620 presso l'editore Ginammi a Venezia Gundulić fa stampare la traduzione dei Salmi di Davide e poi anche altri suoi componimenti. È noto che lo stile letterario di Gundulić si appoggiava da un lato sull'ottima conoscenza dell'italiano e la capacità di tradurre testi letterari dall'italiano nella 
propria madrelingua, e dall'altro sulla conoscenza della tradizione autoctona popolare. Nella prefazione a un'edizione ottocentesca del poema Osman il curatore osserva: "la natura dello spirito slavo gli ha impedito di inciampare nei tranelli della fantasia che si libera della ragione“" (Tkalčević 1854: X). Gundulić sceglie l'ottonario, verso allegro dalla rima facile abbreviando il classico decasillabo narrativo della poesia popolare. È probabile che Gundulić conoscesse la traduzione di Giovanni Andrea dell' Anguillara in ottava rima delle Metamorfosi ovidiane (Venezia 1584) e anche il poema ariostesco. Anche il critico ragusino ottocentesco Francesco Maria Appendini sosteneva che la scelta dell'ottonario era dettata dall'intenzione di esprimersi in maniera schietta e semplice, aggiungendo che il modello italiano si riconosceva nella scelta dei lessemi, più brevi ed eufonici: la lingua di Gundulić era, secondo lui, lirica per eccellenza. Il testo preso in considerazione per questa ricerca è la sua traduzione (o adattamento?) in croato del libretto d'opera dell' Arianna di Ottavio Rinuccini. L'opera viene portata in scena a Mantova il 28 maggio 1608, in occasione delle nozze dell'Infanta Margherita di Savoia e don Francesco Gonzaga, con la musica del maestro di cappella di corte Claudio Monteverdi. Purtroppo, lo spartito è andato perduto e non ci rimane che il famoso Lamento, in alcune varianti create più tardi dallo stesso Monteverdi. Oltre a Mantova, il libretto è stato subito ristampato a Firenze e a Venezia. La protagonista femminile dello spettacolo era l'attrice-cantante Virginia Ramponi, acclamatissima nelle parti patetiche (Bujić 1999: 77). Non sappiamo per quali vie, dirette o indirette, le notizie su quest'opera e lo stesso testo fossero giunti sulla sponda orientale dell'Adriatico. Ma già nel 1615 Gundulić compie l'adattamento dell'opera intitolandolo Arijadna. Il quadro scenico (di otto scene) è una riduzione di genere corrispondente alle preferenze del pubblico nostrano. Di tema pastorale e con lieto fine, probabilmente completata nel 1620, Arijadna è stata rappresentata con successo e pubblicata in seguito dagli amici del poeta ad Ancona presso l'editore Salvioni nel 1633. L'edizione, datata il 4 settembre 1632, reca la dedica all'amico Marinko Tudišević, che probabilmente aveva allestito l'opera, e il titolo: Ariadna tragedia gosp. Giva Frana Gundulica, vlastelina dubrovackoga. Anche se nel 1621 Gundulić aveva definito le proprie opere giovanili "un parto delle tenebre", il titolo e la dedica testimoniano il contrario. Inoltre, nel Seicento i traduttori spesso non davano notizia dell'originale dell'opera tradotta. Secondo alcune ricerche recenti sembra che le arie o improvvisazioni musicali avessero potuto accompagnare il testo sulla scena e che le concatenazioni tra le strofe permettessero di cantare e non solo recitare i versi. Dopo aver esaminato i precedenti contributi al problema sia traduttologico che interculturale, abbiamo messo a confronto i rispettivi testi, italiano e croato del Lamento di Arianna, ossia il frammento più conosciuto del libretto originale, unico che conserva lo spartito musicale monteverdiano (come è noto, le altre parti sono andate perdute) rimarcando i procedimenti traduttivi e gli esiti dell'opera gunduliciana.

\section{Ricerche}

L'Arijadna di Gundulić viene identificata nel volume Notizie storico-critiche sulle antichità, storia e letteratura de'ragusei, (1802) di Francesco Maria Appendini come 
testo originale, e appena nel volume Historia dubrovačke drame (1871) di Armin Pavić viene brevemente riassunta e commentata come traduzione di Rinuccini e vengono riprodotti il testo originale e la traduzione del Lamento. La traduzione viene definita melodrammatica e lirica, ben riuscita e probabilmente musicata, dato che poi risulterà erroneo. Un decennio più tardi Luko Zore pubblica la prima analisi traduttologica dell'Arijadna (Zore 1882), un saggio estremamente pertinente nelle sue osservazioni e conclusioni che lo spazio a disposizione non ci permette di elaborare, ma che merita una riproposta in altra sede. Tra le ricerche più recenti va segnalato il saggio di Nikica Kolumbić del 1977 in cui purtroppo si evince che l'autore non conosce i principi formativi del genere del libretto d'opera, non conosce l'importanza di Rinuccini nella creazione del nuovo genere e non ha le competenze per rilevare le caratteristiche della traduzione né per trattarla come testo derivato e non originale di Gundulić. Al contrario, negli anni novanta i saggi recenti di due illustri musicologi, Bojan Bujić ed Ennio Stipčević, sono serviti a contestualizzare la traduzione e a considerarla in un ambito interdisciplinare, essenziale per confermarne il vero peso letterario. In questo saggio ci limitiamo a indicare e interpretare alcuni esempi tratti dal famoso Lamento con lo scopo di individuare le strategie traduttive usate da Gundulić nella resa dell'originale. Il Lamento occupa i versi 783-864 (del totale di 1115) nel libretto di Rinuccini, mentre nella traduzione si tratta dei versi 1251-1396 (del totale di 1829). Dunque, con un semplice calcolo scopriamo che dagli 81 endecasillabi e settenari dell'originale, nella traduzione siamo arrivati a ben 145 ottonari. In base a dati come questo, i ricercatori hanno spesso concluso che la traduzione fosse in gran parte una creazione d'autore, ma, come vedremo di seguito, si tratta piuttosto di una riproduzione pedissequa del libretto italiano, contrassegnata da quelli che sono dei procedimenti traduttivi molto comuni e frequenti, dell'esplicitazione e della parafrasi, ovvero, nella maggior parte dei casi, dell'adeguamento di un endecasillabo a due ottonari, senza cambiamento del messaggio.

Il primo esempio che proponiamo è l'incipit famoso del Lamento:

Ne ustavlja'te, ne držite,

komu veće nî živiti,

umrit, umrit, jaoh, pustite,

pustite me, jaoh, umriti!

Ter ko će $u$ ovoj hudoj česti

razgovor mi kî donijeti?

U ovoj ljutoj, vaj, bolesti

pustite me, jaoh, umrijeti!
I

I

Lasciatemi morire, lasciatemi morire; e che volete voi che mi conforte in così dura sorte, in così gran martire?

Lasciatemi morire.

Come si nota da subito, Gundulić aggiunge due versi introduttivi impostati grammaticalmente come il seguito, ovvero come una serie di imperativi rivolti a un pubblico (il coro di pescatori) e, dal punto di vista del contenuto, come la stessa preghiera dei primi versi dell'originale resa con motivi antitetici (non fermate, non trattenete chi non ha più voglia di vivere). Nei due versi seguenti osserviamo un'aggiunta dell'interiezione jaoh (ahimè) senza valore semantico, 
che è presente in tutto il dramma come espediente per rimediare al numero di sillabe necessario. Vediamo inoltre che la ripetizione anaforica dell'originale si trasforma in chiasmo. Nei prossimi quattro versi si nota una lieve modifica della struttura sintattica in cui il penultimo verso non è più parte della frase precedente, ma si allaccia artificialmente alla ripresa del messaggio iniziale. Anche se i motivi originali rimangono inalterati (bolest - martire), il significato e lo stile sono cambiati ed è stata eliminata anche la seconda anafora (in così...). Già da questo breve esempio è chiaro che la versione croata non poteva servire come libretto, sicuramente non per la musica di Monteverdi.

Nel secondo esempio vediamo un maggior discostamento dal significato originale, pur usando lo stesso motivo degli occhi che propone l'originale:

O Tezeo, moj Tezeo, moj, er moj si, jaoh, svakako, zasve očima mojim tako

Svitlos dragu da si uzeo.
O Teseo, o Teseo mio, sì che mio ti vo' dir, che mio pur sei, benché t'involi, ahi crudo! a gl'occhi miei.

Dall'iniziale significato di "benché sei fuggito dalla mia vista" otteniamo nella traduzione una versione più lirica: "anche se hai rubato ai miei occhi la cara luce" e l'omissione dell'importante denuncia di crudeltà. I quattro versi citati (12631266) verranno ripetuti tali e quali ancora una volta nella traduzione (1351-1354) senza motivazione nell'originale. Il terzo esempio che viene proposto dimostra come Gundulić abbia fatto attenzione a riprodurre lo stile dell'originale:

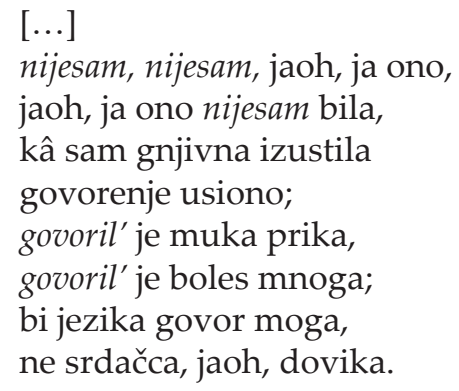

non son, non son quell'io, non son quell'io che i feri detti sciolse:

parlò l'affanno mio, parlò il dolore; parlò la lingua sì, ma non già 'l core.

Nuovamente abbiamo nei primi due versi un tipo di chiasmo intercalato dall'interiezione, ma si nota che ci sono esattamente tre ripetizioni dell'originale "non son". Il terzo settenario viene amplificato a ben due ottonari con l'aggiunta dell'aggettivo gnjiona - arrabbiata e con la traduzione letterale dei "feri detti" govorenje usiono, che però in croato ammonta al doppio delle sillabe, creando un verso autonomo. La seconda parte dell'esempio si basa sulla triplice ripetizione anaforica del verbo "parlò" seguito dall'oggetto diretto che nella traduzione si limita a due riprese e all'amplificazione dell'oggetto. L'ultimo verso della traduzione rimedia alle sillabe usando il diminutivo di "core" e l'interiezione jaoh. Bisogna sottolineare che in questo esempio Gundulić realizza anche un buon ritmo declamatorio.

L'ultimo esempio è stato scelto per corroborare la tesi dell'amplificazione decorativa e non contenutistica dell'originale: 
Ah Tezeo, moj Tezeo, skončat li ćeš u nemiru onu ku si ti na viru iz rodnoga mjesta izveo? Ah, žuđeni moj pokoju, ah, životu moj izbrani, podnijet li ćeš da zamani plačuć pitam pomoć tvoju?

Ah, sve dobro mêpod nebi, brače, ter ćeš ti pustiti

Arijadnu tužnu umriti, slavu i život ka da tebi?
Ah Teseo, ah Teseo mio, lascerai tu morire,

in van piangendo, in van gridando aita

la misera Arianna, che a te fidossi e ti diè gloria e vita?

Dei sei versi di Rinuccini, articolati come una proposizione interrogativa, come un unico pensiero, nella traduzione troviamo esattamente il doppio dei versi, dodici ottonari, e ben tre proposizioni interrogative. Le aggiunte, demarcate in corsivo, sono ripetizioni di fatti conosciuti (lascerai morire colei che in buona fede portasti via dalla patria) e invocazioni metaforiche dell'amato modellate sul primo verso (ah, bramato mio conforto / ah, vita mia che io sola scelsi / ah, tutto il bene mio sotto il cielo). Dunque, vengono aggiunti sfoghi sentimentali tipicamente barocchi, inesistenti nel prototesto, che non contengono niente di nuovo dal punto di vista del messaggio poetico.

\section{Conclusione}

Rivalutando le ricerche precedenti è lecito dire che Kolumbić nel suo saggio pecca di alcuni errori fondamentali. Innanzitutto, valuta la traduzione come se si trattasse di un testo d'autore, come se Gundulić avesse volontariamente creato la caratterizzazione dei personaggi, come se fossero sue le scelte drammaturgiche nella traduzione. Nel suo saggio propone (come abbiamo fatto anche noi) degli esempi con il testo a fronte, ma non sa qualificarne la dimensione traduttologica e si limita a constatare che Gundulić abbia aggiunto del materiale, ritenendo questo intervento bastante per conferirgli la prevalente autorialità del pezzo. Quello che è veramente un intervento d'autore, che viene sottolineato soltanto nelle ricerche di Bojan Bujić, è la suddivisione formale del testo nei cinque atti in cui lo troviamo stampato nel 1633. Infatti, l'atto unico di Rinuccini non avrà nemmeno la suddivisione in scene, che viene adottata soltanto nelle ristampe consecutive, per facilitare la lettura. Il saggio di Kolumbić è informativo dal punto di vista della datazione, ha ragione a concludere che queste traduzioni che hanno avviato la carriera teatrale di Gundulić sono state fondamentali per la sua crescita e disciplina versificatoria nelle sue opere autoriali, ma purtroppo sottovaluta (come si sottovalutava negli anni '70 del secolo scorso) la librettistica come nuovo genere letterario, diverso dalla pastorale e dalla tragedia parlata, e soprattutto sottovaluta l'importanza e il valore artistico e storico-musicale/letterario di Rinuccini. Vent'anni più tardi, anche Stipčević fa un errore di interpretazione constatando che Gundulić non abbia 
solo tradotto ma abbia seguito una logica poetica personale (Stipčević 1994: 185) mentre abbiamo visto dagli esempi che si tratta di una traduzione pedissequa, ma abbondante di aggiunte tipicamente barocche, di interiezioni volte a completare la quantità del verso, di invocazioni convenzionali, ecc. che però non si discostano mai dalla trama e dall'idea del prototesto. Neanche a ripetere che non avviene nessun cambiamento di caratterizzazione, di ambientazione, di elementi specifici. Ritorniamo alla conclusione, che solo l'analisi di Zore del 1882 coglie e propone perspicacemente le strategie traduttive applicate, ne dà degli esempi pertinenti, spiega magnificamente perché è sbagliato dal punto di vista culturologico tradurre Alceste come Ljubmir (Zore 1882: 188-189) e ci informa che l'introduzione dei due personaggi dei pescatori - corifei, inesistenti in Rinuccini, è l'unico intervento originale di Gundulić. Al termine della nostra analisi possiamo constatare che questo tema non è affatto stato esaurito, che merita uno scrutinio più elaborato, corroborato da altri esempi, non solo quelli del Lamento. Da questo breve percorso concludiamo che Gundulić nella sua traduzione usa tutti i procedimenti traduttivi legati all'amplificazione del testo: la moltiplicazione di elementi, la parafrasi, l'aggiunta d'autore, l'esplicazione, ma anche (paradossalmente) l'omissione. Molte delle sue soluzioni traduttive, che considerate approssimativamente possono sembrare adeguate, nascondono invece errori di interpretazione del prototesto, oppure riprese di motivi identici, ma con significati diversi. Abbiamo però visto anche una grande cura dedicata allo stile, al ritmo del verso, soprattutto alla resa degli effetti fonici dell'originale e siamo dell'opinione che l'Arijadna rappresenta un ottimo esempio di traduzione e adattamento in cui il prodotto finale assume nuove valenze nell'ambito della cultura ricevente.

\section{Bibliografia}

Bujić, Bojan (1998). Pastorale o melodramma? Le traduzioni croate di Euridice e Arianna per le scene di Dubrovnik, in: Musica e storia, VI/2, pp. 477-499.

Bujić, Bojan (1999). Rinuccini the Craftsmen: A View of his L'Arianna, in: Early Music History, vol. 18, pp. 75-117.

Gundulić, Ivan (1938). Arijadna, in: Djela Giva [Dživa] Frana Gundulića, a cura di Đuro Körbler, rivisto da Milan Rešetar, Zagreb, JAZU, pp. 137-197.

Kolumbić, Nikica (1977). Gundulićeve rane drame i formiranje njegove pjesničke ličnosti, in: Dani Hvarskoga kazališta, vol. 4, 1, pp. 51-76.

Rinuccini, Ottavio. Lamento di Arianna, in: Fabbri, Paolo (2003). Il secolo cantante, Roma, Bulzoni, pp. 28-29.

Stipčević, Ennio (1994). »Skladno pripjevanje«: teatar, glazba i glazbeni teatar, in: Dani Hvarskoga kazališta, a cura di Nikola Batušić et al., Split, Književni krug, pp. 175-194.

Tkalčević, Adolf Veber (1854), Ivan Franjin Gundulić in: Gundulić, Ivan, Osman u dvadeset pievanjah, Zagreb, Matica ilirska, pp. III-XXIX.

Zore, Luko (1882) Gragja za književno-povijesnu ocjenu Gundulićeve "Arijadne", rad JAZU, pp. 129-189. 


\section{Gundulić prevoditelj Rinuccinija. Analiza hrvatske inačice Arijadnine tužaljke}

U radu se analizira hrvatski prijevod libreta Arianna, talijanskoga pjesnika Ottavia Rinuccinija, koji slavu duguje uglazbitelju, prvome značajnom opernom skladatelju, Claudiju Monteverdiju. Opera je prvi put izvedena 1608., a već oko 1615. tekst je izveden u Dubrovniku na hrvatskom kao govorena drama, u prijevodu Ivana Gundulića. Prepjev je objavljen 1633. i djelo je jednoga od najznačajnijih hrvatskih baroknih autora koji se u svojoj književnoj karijeri višestruko pozabavio prijevodima s talijanskoga. U istraživanju se usporedno analizira izvornik i prijevod čuvene Arijadnine tužaljke (Lamento di Arianna), najpoznatijega ulomka iz libreta zato što je jedini za koji imamo sačuvanu Monteverdijevu glazbenu partituru, dok je ostatak izgubljen. Pomoću nekoliko primjera predstavljaju se neki Gundulićevi specifični leksički i stilistički odabiri i utvrđuje se kojim se prijevodnim postupcima poslužio kako bi tekst prilagodio odabranoj versifikaciji i drugačijoj dramskoj izvedbi u odnosu na izvornik.

Ključne riječi: Gundulić, Rinuccini, Arianna, prijevod, Zore 
